\title{
Finding the optimal cutoff value for amyloid $\beta$ positivity using the iterative outlier method and concordance rate
}

\author{
Hang-Rai Kim ${ }^{1,2,3,4,}$, , Yeong Sim Choe ${ }^{1,2,3,5,}$, Seung Hwan Moon ${ }^{6}$, Hee Jin Kim ${ }^{1,2,3,5}$, \\ Hyemin Jang ${ }^{1,2,3}$, Duk L.Na ${ }^{1,2,3,5,7}$, Seongbeom Park ${ }^{8}$, Sang Won Seo S,2,3,5,9,10 $^{1}$
}

${ }^{1}$ Department of Neurology, Samsung Medical Center, Sungkyunkwan University School of Medicine, Seoul, Korea

${ }^{2}$ Neuroscience Center, Samsung Medical Center, Seoul, Korea

${ }^{3}$ Samsung Alzheimer Research Center, Samsung Medical Center, Seoul, Korea

${ }^{4}$ Department of Neurology, Dongguk University Ilsan Hospital, Goyang, Korea

${ }^{5}$ Department of Health Sciences and Technology, Samsung Advanced Institute for Health Sciences \& Technology, Sungkyunkwan University, Seoul, Korea

${ }^{6}$ Department of Nuclear Medicine, Samsung Medical Center, Sungkyunkwan University School of Medicine, Seoul, Korea

${ }^{7}$ Stem Cell \& Regenerative Medicine Institute, Samsung Medical Center, Seoul, Korea

${ }^{8}$ Heuron Co. Ltd., Incheon, Korea

${ }^{9}$ Department of Clinical Research Design \& Evaluation, Samsung Advanced Institute for Health Sciences \& Technology,

Sungkyunkwan University, Seoul, Korea

${ }^{10}$ Center for Clinical Epidemiology, Samsung Medical Center, Seoul, Korea

Received: January 25, 2021

Revised: April 27, 2021

Accepted: May 3, 2021

Corresponding author:

Sang Won Seo

Department of Neurology,

Samsung Medical Center,

Sungkyunkwan University

School of Medicine, 81 Irwon-

ro, Gangnam-gu, Seoul 06351,

Korea

Tel: +82-2-3410-1233

E-mail:

sangwonseo@empal.com

${ }^{\star}$ Hang-Rai Kim and Yeong Sim Choe contributed equally to this study as first authors

This is an Open Access article distributed under the terms of the Creative Commons Attribution Non-Commercial License (https:// creativecommons.org/licenses/ by-nc/4.0/).

\section{ABSTRACT}

Purpose: The purpose of this study was to calculate the cutoff value for amyloid $\beta$ ( $A \beta$ ) positron emission tomography (PET) positivity using the iterative outlier method and to evaluate its validity based on the concordance rate.

Methods: We performed the iterative outlier method on 373 cognitively unimpaired (CU) subjects and calculated the optimal cutoff value for $A \beta$ positivity. The validation was performed using the independent dataset, comprising 83 subjects ( $27 \mathrm{CU}, 27 \mathrm{am}$ nestic mild cognitive impairment, and 29 Alzheimer's dementia). We evaluated the validity of the $A \beta$ cutoff value by calculating its concordance rate with the visual assessment and between two different $A \beta$ tracers performed in the same subject.

Results: The concordance rate of $A \beta$ cutoff values with the visual assessment ranged from $84.3 \%$ to $91.5 \%$, depending on the reference regions. The concordance rate of the cutoff values between the two $A \beta$ tracers ranged from $90.3 \%$ to $97.5 \%$, all of which were higher than that of the visual assessment (86.7\%).

Conclusion: We demonstrated that the iterative outlier method could identify the cutoff value for $A \beta$ PET positivity.

Keywords: Alzheimer disease; Amyloid; Positron-emission tomography 
PRECISION AND FUTURE MIEDICINE

Optimal cutoff value for amyloid $\beta$ positivity

\section{INTRODUCTION}

Alzheimer's disease (AD) is the most common cause of dementia in the elderly [1], and is characterized by the deposition of amyloid $\beta(A \beta)$ in the brain. The amyloid cascade hypothesis, a well-known hypothetical model of $A D$, posits that accumulation of $A \beta$ is the earliest pathogenic process leading to tau deposition, neurodegeneration, and clinical cognitive impairment [2]. Therefore, distinguishing brains free of $A \beta$ from those with $A \beta$ deposition is of utmost importance for the early diagnosis of $A D[3]$.

$A \beta$ deposition in the brain can be measured directly by brain autopsy or indirectly by either cerebrospinal fluid (CSF) sampling or $A \beta$ tracing positron emission tomography (PET) [4]. A $\beta$ PET is more advantageous than CSF sampling as it is less invasive and detects regional $A \beta$ depositions. Measurement of $A \beta$ deposition by PET is by either visual assessment or more objective measures, such as by calculating the standard uptake value ratio (SUVR) [5] or distribution volume ratio (DVR) [6]. However, since both SUVR and DVR are continuous measures, it is necessary to dichotomize them into evidence (positive) or no evidence of deposition (negative).

Previous studies have presented a variety of approaches to define $A \beta$-positive cutoffs, including the iterative outlier method [7]. Until now, the validity of these cutoffs has often been evaluated with the standard-of-truth (SOT) based on either histological findings of brain autopsy or visual assessment of PET images. However, visual assessment is operator-dependent [8] and it is difficult to obtain postmortem data.

In this study, we calculated the cutoff value for $A \beta$ positivity using the iterative outlier method and evaluated its validity based on the concordance rate. Two tracers were used to evaluate the concordance rate of $A \beta$ positivity based on the cutoff value in the same subject.

\section{METHODS}

\section{Development dataset}

For the iterative outlier method, we used A $\beta$ PET data obtained from 373 cognitively unimpaired (CU) subjects aged over 50 years. CU subjects were defined as those with normal results on neurological examination and normal cognitive function, expected for their ages and education, on a standardized cognitive test (Seoul Neuropsychological Screening Battery) [9]. Of the 373 subjects, 202 and 171 subjects underwent ${ }^{18} \mathrm{~F}$-florbetaben (FBB) and ${ }^{18} \mathrm{~F}$-flutemetamol (FMM) $A \beta$
PET, respectively.

\section{Validation dataset}

For validation of the $A \beta$-positive cutoff value, we used the head-to-head comparison data, comprising 83 subjects ( 27 CU, 27 amnestic mild cognitive impairment, and $29 \mathrm{AD}$ ), in whom both FBB and FMM AB PET were performed. A detailed description is provided in our previous study [10].

\section{Magnetic resonance imaging acquisition}

Standardized three-dimensional T1 turbo field echo images were acquired from all subjects at the Samsung Medical Center using the same 3.0-T magnetic resonance imaging (MRI) scanner (Philips Achieva, Philips Healthcare, Andover, MA, USA). The following parameters were used: sagittal slice thickness of $1.0 \mathrm{~mm}$, over contiguous slices with $50 \%$ overlap, no gap, repetition time of $9.9 \mathrm{~ms}$, echo time of $4.6 \mathrm{~ms}$, flip angle of $8^{\circ}$, and matrix size of $240 \times 240$ pixels, reconstructed to $480 \times 480$ over a field of view of $240 \mathrm{~mm}$.

\section{PET acquisition}

PET images were acquired using a Discovery STe PET/computed tomography (CT) scanner (GE Medical Systems, Milwaukee, WI, USA) in three-dimensional scanning mode that examined 47 slices of 3.3-mm thickness spanning the entire brain. CT images were acquired using a 16-slice helical CT (140 KeV, 80 mA; 3.75-mm section width) for attenuation correction. According to protocols proposed by the ligand manufacturers, a 20-minute emission PET scan with dynamic mode (consisting of $4 \times 5$ minutes frames) was performed 90 minutes after injection of a mean dose of $311.5 \mathrm{MBq}$ FBB and 185 MBq FMM, respectively. Three-dimensional PET images were reconstructed in a $128 \times 128 \times 48$ matrix with a voxel size of $2 \times 2 \times 3.27 \mathrm{~mm}$ using the ordered-subsets expectation maximization algorithm (FBB iterations $=4$ and subset $=$ 20; FMM iterations $=4$ and subset $=20$ ). $A \beta$ positivity was determined based on visual assessments performed by three experienced raters (two nuclear medicine doctors and one neurologist) [11-15].

\section{Image preprocessing and calculation of the SUVR}

To calculate the SUVR, we performed the following processes. First, we co-registered the PET image to the T1-MRI images. Subsequently, we normalized the native PET image to the Montreal Neurological Institute-152 template using the transformation matrix, calculated during the segmentation of T1MRI. After normalization, the brain was divided into 116 grey 
matter regions using the automated anatomical labeling (AAL) atlas [16]. We considered three reference regions (whole cerebellum [WC], cerebellar gray matter [CG], and pons) to calculate the SUVR, the regional masks of which were obtained from the GAAIN website (http://www.GAAIN.org) [17]. We calculated the global cortical SUVR as the volume-weighted mean of 56 cortical regions in the AAL template [18]. This pre-processing was performed using SPM8 through Matlab 2014b (Mathworks, Natick, MA, USA).

\section{Iterative outlier method}

We used the iterative outlier method and generated an upper- and lower-bound SUVR. During the iteration, subjects with greater than the upper inner-bound (3rd quartile +1.5 interquartile range [IQR] or less than the lower inner-bound [1st quartile-1.5 IQR]) values were removed from the dataset. This process was repeated until all outliers were removed. In the final dataset, a cutoff value was determined by adding $2.5 \%$ of the maximum SUVR in itself [19].

\section{Statistical analysis}

We performed two analyses using the independent dataset to validate the $A \beta$ cutoff value. We first compared the cutoff value-derived $A \beta$ positivity with the visually determined $A \beta$ positivity and calculated the concordance rate. Next, we evaluated whether the cutoff value-derived $A \beta$ positivity of one subject was concordant between the two $A \beta$ tracers (FBB and FMM). We assumed that the optimal cutoff value would distinguish subjects with positive $A \beta$ deposition regardless of the tracer type. The statistical significance of the concordance rate was evaluated using the McNemar's chisquare test, with a P-value of less than 0.05 indicating non- agreement between the two measures. All statistical analyses were completed using R version 3.5.3 (http://www.r-project.org/). This is a retrospective study using the data from the preceding study [10], which was approved by the Institutional Review Board of Samsung Medical Center (2015-04091) with written informed consent of the participants at the time of enrollment.

\section{RESULTS}

\section{Subject demographics}

Table 1 shows the demographics of the development and validation datasets. In the development dataset, subjects who underwent FBB were older than those who underwent FMM. In the validation dataset, although two different $A \beta$ PETs were performed in the same subject, the frequency of $A$ $\beta$ positivity differed between the two tracers.

\section{$A \beta$ cutoff value with the iterative outlier method} Using the iterative outlier method, we calculated the optimal cutoff value for $A \beta$ PET positivity according to the $A \beta$ tracers and reference regions (Fig. 1). In both FBB and FMM, the cutoff value was higher when the CG was used as the reference region.

\section{Validation of the cutoff value based on the concordance rate with the visual assessment}

To validate the cutoff value derived from the iterative outlier method, we applied the cutoff value for $A \beta$ positivity in the independent dataset and compared it with that of the visual assessment (Table 2). The concordance rate ranged from $84.3 \%$ to $91.5 \%$, and the highest rate was observed when the

Table 1. Demographics of the development and validation datasets

\begin{tabular}{|c|c|c|c|c|}
\hline \multirow{2}{*}{ Demographic } & \multicolumn{2}{|c|}{ Development dataset } & \multicolumn{2}{|c|}{ Validation dataset } \\
\hline & $\operatorname{FBB}(n=202)$ & $\operatorname{FMM}(n=171)$ & FBB $(n=83)$ & $\operatorname{FMM}(n=83)$ \\
\hline Age (yr) & $71.4 \pm 7.2$ & $68.7 \pm 7.4$ & \multicolumn{2}{|c|}{$71.2 \pm 7.7$} \\
\hline Women & $118(58.4)$ & $109(63.7)$ & \multicolumn{2}{|c|}{$50(60.2)$} \\
\hline Education (yr) & $12.6 \pm 4.4$ & $11.6 \pm 5.0$ & \multicolumn{2}{|c|}{$9.8 \pm 4.8$} \\
\hline MMSE, score & $28.3 \pm 1.5$ & $28.0 \pm 2.1$ & \multicolumn{2}{|c|}{$25.5 \pm 5.0$} \\
\hline Diagnosis (CU/aMCI/AD) & $202 / 0 / 0$ & $171 / 0 / 0$ & \multicolumn{2}{|c|}{$27 / 27 / 29$} \\
\hline$A \beta$ positivity ${ }^{a)}$ & $32(15.8)$ & $27(15.8)$ & $45(54.2)$ & $52(62.7)$ \\
\hline
\end{tabular}

Values are presented as mean \pm standard deviation or number (\%).

$\mathrm{FBB},{ }^{18} \mathrm{~F}$-florbetaben; FMM, ${ }^{18} \mathrm{~F}$-flutemetamol; MMSE, Mini-Mental State Examination; CU, cognitive unimpaired; aMCl, amnestic mild cognitive impairment; $A D$, Alzheimer's disease; $A \beta$, amyloid $\beta$.

${ }^{a)} A \beta$ positivity was determined based on visual assessments. 
PRECISION AND FUTURE MEDICINE

Optimal cutoff value for amyloid $\beta$ positivity

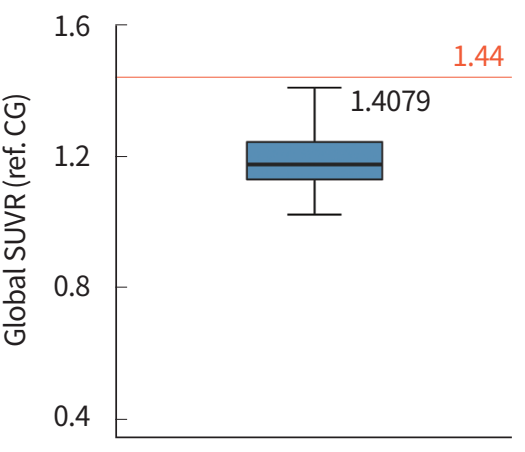

(A)

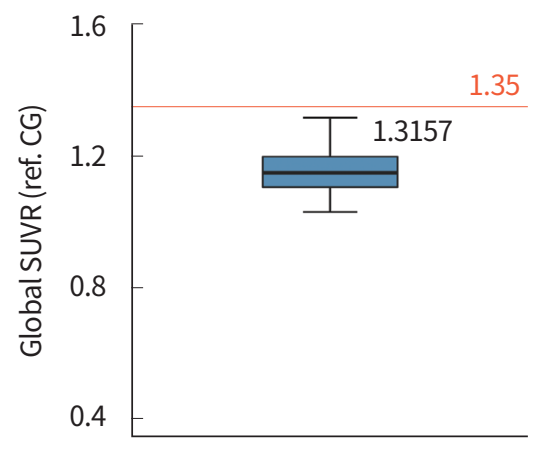

FBB

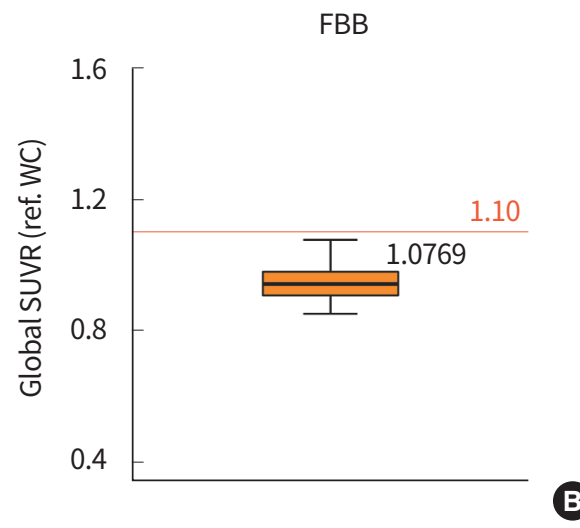

FMM

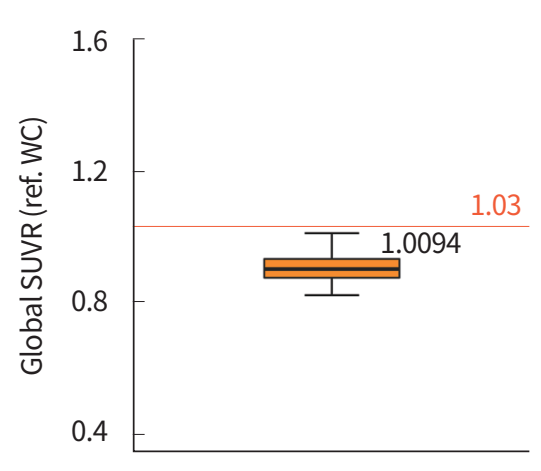

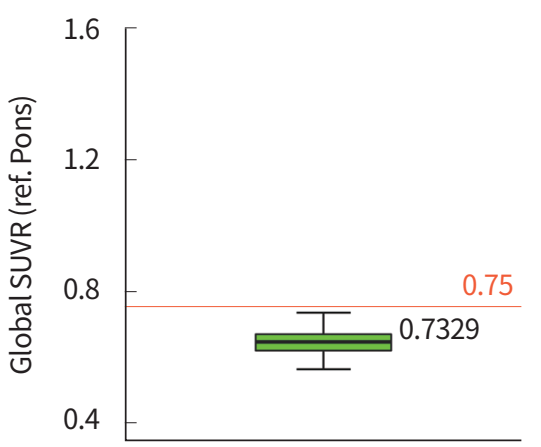

C

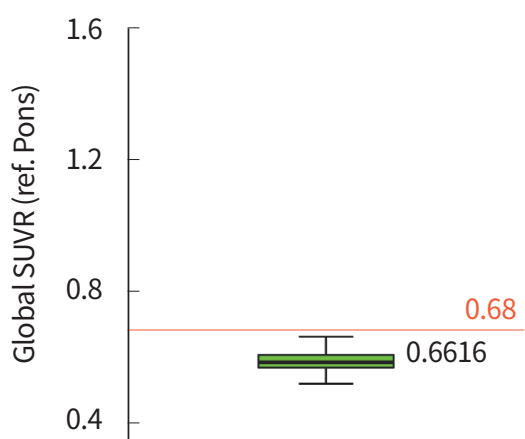

F

Fig. 1. Results of the iterative outlier method for (A, B, C ${ }^{18} \mathrm{~F}$-florbetaben (FBB) and (D, E, F) ${ }^{18} \mathrm{~F}$-flutemetamol (FMM). Red lines indicate cutoff value derived using the iterative outlier method. SUVR, standard uptake value ratio; CG, cerebellar gray matter; WC, whole cerebellum.

Table 2. The concordance rate of the cutoff value with visual assessment

\begin{tabular}{lccc}
\hline A $\beta$ tracer & $\begin{array}{c}\text { Reference } \\
\text { region }\end{array}$ & $\begin{array}{c}\text { Concordance } \\
\text { rate }\end{array}$ & P-value $^{\text {a) }}$ \\
\hline FBB & CG & 0.855 & 0.773 \\
& WC & 0.915 & 0.450 \\
FMM & Pons & 0.903 & 0.724 \\
& CG & 0.843 & 0.579 \\
& WC & 0.855 & 0.773 \\
& Pons & 0.843 & 0.579
\end{tabular}

$A \beta$, amyloid $\beta ; F B B,{ }^{18}$ F-florbetaben; $C G$, cerebellar gray matter; $W C$, whole cerebellum; FMM, ${ }^{18} \mathrm{~F}$-flutemetamol.

${ }^{\text {a) }}$-value was calculated using McNemar's chi-square test.

WC was used as the reference for both FBB and FMM. All $P$-values were greater than 0.05 , indicating that the two measures (the cutoff value and the visual assessment) were in accordance.

\section{Validation of the cutoff value based on the concordance rate between FBB and FMM}

We evaluated whether the cutoff value-derived $A \beta$ positivity
Table 3. The concordance rate of the SUVR cutoff between the two $A \beta$ tracers

\begin{tabular}{lcc}
\hline Methods & Concordance rate & P-value $^{\text {a) }}$ \\
\hline SUVR cutoff & & \\
CG & 0.903 & 0.077 \\
WC & 0.975 & 0.479 \\
Pons & 0.975 & 0.479 \\
Visual assessment & 0.867 & 0.070 \\
\hline
\end{tabular}

SUVR, standard uptake value ratio; $A \beta$, amyloid $\beta$; CG, cerebellar gray matter; WC, whole cerebellum.

${ }^{a} \mathrm{P}$-value was calculated using McNemar's chi-square test.

was consistent between the two tracers. The concordance rate ranged from $90.3 \%$ to $97.5 \%$, all of which were higher than that of the visual assessment (86.7\%).

The concordance rate of visual assessment between FBB and FMM was $86.7 \%$ (72/83) (Table 3, Fig. 2). There were 11 subjects who showed discordant results (nine subjects [FBB negative/FMM positive] and two subjects [FBB positive/FMM negative]). The concordance rates of the cutoff values were $90.3 \%$ (75/83), $97.5 \%$ (81/83), and $97.5 \%$ (81/83) for each reference region (CG, WC, and pons, respectively) (Table 3). The 
PRECISION AND FUTURE MEDICINE

Hang-Rai Kim, et al.
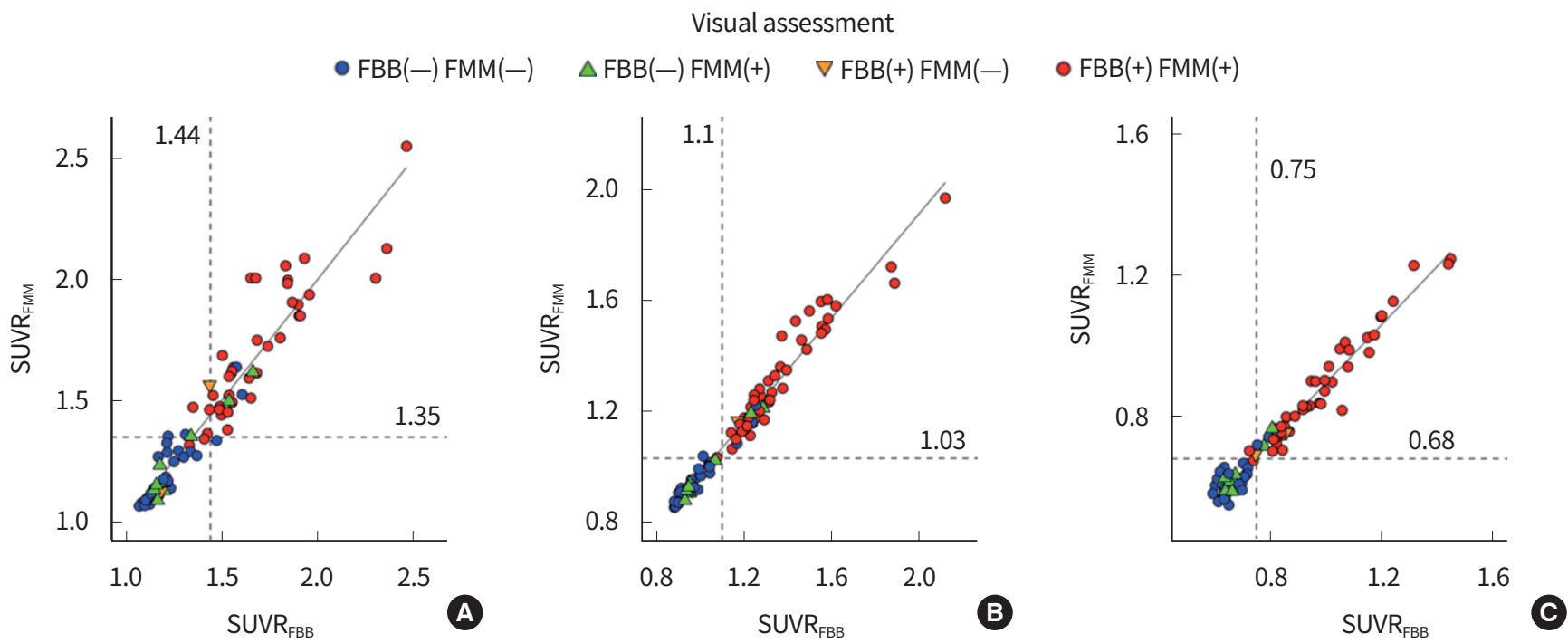

Fig. 2. Scatter plot of ${ }^{18} \mathrm{~F}$-florbetaben (FBB) and ${ }^{18} \mathrm{~F}$-flutemetamol (FMM) standard uptake value ratio (SUVR) with (A) cerebellar gray matter, (B) whole cerebellum, and (C) pons as the reference regions. Dotted lines indicate the cutoff value.

majority of the subjects who showed discordant results in the visual assessment showed concordant results when the cutoff value was used (9/11 for the CG [Fig. 2A], 11/11 for the WC [Fig. 2B], and 10/11 for the pons [Fig. 2C]).

\section{DISCUSSION}

In this study, we identified the optimal SUVR cutoff for $A \beta$ positivity using the iterative outlier method and evaluated its validity based on the concordance rate.

We found that the cutoff optimization methods were comparable to visual assessment. Detecting individuals with $A B$ deposition is important, as it is valuable for the clinical diagnosis of $A D$ and in clinical trials aiming to reduce the burden of $A \beta$ in the brain [4]. To date, in the clinical field, $A \beta$ positivity of PET is generally determined by experienced clinicians based on visual rating. However, visual rating can be operator- and $A \beta$ tracer-dependent, thus are prone to intra- and inter-rater discrepancies [8]. Interestingly, we found that the iterative outlier method-derived cutoff value had a higher concordance rate of $A \beta$ positivity between the two tracers than that of the visual assessment. Although its superiority to visual assessment should be tested statistically, this finding suggests that the iterative outlier method-derived cutoff value could be an accurate measure of $A \beta$ positivity in FBB and FMM PET.

Although the iterative outlier method may have an advantage over visual assessment, there are other approaches to define $A \beta$-positive cutoff values, such as receiver operating characteristic (ROC) analyses [20] and clustering methods [21]. The important difference in the approaches is that the iterative outlier method requires an annotated group of cognitively healthy subjects [7], whereas the ROC method requires both diseased and healthy groups [20], and the cluster analysis requires mixed groups that do not require annotations [21].

We further observed that cutoff optimization methods differed according to the reference regions and types of $A \beta$ tracers. This discrepancy precluded the generalization of findings from one $A \beta$ tracer to another [22]. Therefore, studies based on $A \beta$ PET data should describe how the data is processed along with a description of the reference regions and the type of tracer.

The strengths of this study are that we used a data-driven approach to identify cutoff values and used a unique cohort (head-to-head comparison data) for the validation, where two different types of A $\beta$ PET were performed in the same subject. However, this study has several limitations. First, we did not perform a statistical test to compare newly derived cutoff values with results of the visual assessment. This can be done by the bootstrapping method; however, the small sample size of our study precluded this analysis. Second, the iterative outlier method depends on the dataset used. Although we tried to obtain a homogenous dataset by thorough examinations, it is possible that the cutoff value will change if more subjects are included. Therefore, our findings should be interpreted with caution and replicated using a larger dataset. Lastly, ideally, histopathological confirmation 
of $A \beta$ deposition in the brain should be the SOT. Due to the scarcity of post-mortem data, we considered visual assessment of $A B$ PET as the SOT in this study.

In conclusion, we demonstrated that the iterative outlier method could identify the cutoff value for $A \beta$ positivity in both FBB and FMM PET.

\section{CONFLICTS OF INTEREST}

No potential conflict of interest relevant to this article was reported. Sang Won Seo has been editorial board of P\&FM since December 2017. He was not involved in the review process of this article.

\section{ORCID}

Hang-Rai Kim

Yeong Sim Choe

Seung Hwan Moon

Hee Jin Kim

Hyemin Jang

Duk L. Na

Seongbeom Park

Sang Won Seo https://orcid.org/0000-0003-4197-4541 https://orcid.org/0000-0001-8921-749X https://orcid.org/0000-0003-4302-1920 https://orcid.org/0000-0002-3186-9441 https://orcid.org/0000-0003-3152-1274 https://orcid.org/0000-0002-0098-7592 https://orcid.org/0000-0002-0759-6826 https://orcid.org/0000-0002-8747-0122

\section{AUTHOR CONTRIBUTIONS}

Conception or design: HRK, YSC, SWS.

Acquisition, analysis, or interpretation of data: HRK, YSC, SHM, HJK, HJ, DLN, SP, SWS.

Drafting the work or revision: HRK, YSC, SWS.

Final approval of the manuscript: HRK, YSC, SWS.

\section{REFERENCES}

1. Alzheimer's Association. 2018 Alzheimer's disease facts and figures. Alzheimers Dement 2018;14:367-429.

2. Jack CR Jr, Knopman DS, Jagust WJ, Petersen RC, Weiner MW, Aisen PS, et al. Tracking pathophysiological processes in Alzheimer's disease: an updated hypothetical model of dynamic biomarkers. Lancet Neurol 2013;12:207-16.

3. Sperling RA, Aisen PS, Beckett LA, Bennett DA, Craft S, Fagan AM, et al. Toward defining the preclinical stages of Alzheimer's disease: recommendations from the National Institute on Aging-Alzheimer's Association workgroups on diagnostic guidelines for Alzheimer's disease. Alzheimers Dement 2011;7:280-92.
4. Blennow K, Mattsson N, Scholl M, Hansson O, Zetterberg $\mathrm{H}$. Amyloid biomarkers in Alzheimer's disease. Trends Pharmacol Sci 2015;36:297-309.

5. McNamee RL, Yee SH, Price JC, Klunk WE, Rosario B, Weissfeld $\mathrm{L}$, et al. Consideration of optimal time window for Pittsburgh compound B PET summed uptake measurements. J Nucl Med 2009;50:348-55.

6. Logan J, Fowler JS, Volkow ND, Wang GJ, Ding YS, Alexoff DL. Distribution volume ratios without blood sampling from graphical analysis of PET data. J Cereb Blood Flow Metab 1996;16:834-40.

7. Aizenstein HJ, Nebes RD, Saxton JA, Price JC, Mathis CA, Tsopelas ND, et al. Frequent amyloid deposition without significant cognitive impairment among the elderly. Arch Neurol 2008;65:1509-17.

8. Haller S, Montandon ML, Lilja J, Rodriguez C, Garibotto V, Herrmann FR, et al. PET amyloid in normal aging: direct comparison of visual and automatic processing methods. Sci Rep 2020;10:16665.

9. Kang Y, Na D, Hahn S. Seoul neuropsychological screening battery. Incheon (KR): Human brain research \& consulting Co.; 2003.

10. Cho SH, Choe YS, Kim YJ, Kim HJ, Jang H, Kim Y, et al. Head-to-head comparison of 18F-florbetaben and 18Fflutemetamol in the cortical and striatal regions. J Alzheimers Dis 2020;76:281-90.

11. Clark CM, Schneider JA, Bedell BJ, Beach TG, Bilker WB, Mintun MA, et al. Use of florbetapir-PET for imaging beta-amyloid pathology. JAMA 2011;305:275-83.

12. Farrar G. Regional visual read inspection of [18F] flutemetamol brain images from end-of-life and amnestic $\mathrm{MCl}$ subjects. J Nucl Med 2017;58(Suppl 1):1250.

13. Johnson KA, Sperling RA, Gidicsin CM, Carmasin JS, Maye $\mathrm{JE}$, Coleman RE, et al. Florbetapir (F18-AV-45) PET to assess amyloid burden in Alzheimer's disease dementia, mild cognitive impairment, and normal aging. Alzheimers Dement 2013;9(5 Suppl):S72-83.

14. Kim HJ, Cho H, Werring DJ, Jang YK, Kim YJ, Lee JS, et al. 18F-AV-1451 PET imaging in three patients with probable cerebral amyloid angiopathy. J Alzheimers Dis 2017;57: 711-6.

15. Martinez G, Flicker L, Vernooij RW, Fuentes Padilla P, Zamora J, Roque i Figuls M, et al. F-18 PET ligands for the early diagnosis of Alzheimer's disease dementia and other dementias in people with mild cognitive impairment (MCI). Cochrane Database Syst Rev 2016;(5):CD012216.

16. Tzourio-Mazoyer N, Landeau B, Papathanassiou D, Crivel- 
lo F, Etard O, Delcroix N, et al. Automated anatomical labeling of activations in SPM using a macroscopic anatomical parcellation of the MNI MRI single-subject brain. Neuroimage 2002;15:273-89.

17. Klunk WE, Koeppe RA, Price JC, Benzinger TL, Devous MD Sr, Jagust WJ, et al. The Centiloid Project: standardizing quantitative amyloid plaque estimation by PET. Alzheimers Dement 2015;11:1-15.

18. Park JH, Seo SW, Kim C, Kim SH, Kim GH, Kim ST, et al. Effects of cerebrovascular disease and amyloid beta burden on cognition in subjects with subcortical vascular cognitive impairment. Neurobiol Aging 2014;35:254-60.

19. Mormino EC, Brandel MG, Madison CM, Rabinovici GD, Marks S, Baker SL, et al. Not quite PIB-positive, not quite PIB-negative: slight PIB elevations in elderly normal control subjects are biologically relevant. Neuroimage 2012;
59:1152-60

20. Devanand DP, Mikhno A, Pelton GH, Cuasay K, Pradhaban $G$, Dileep Kumar JS, et al. Pittsburgh compound B (11CPIB) and fluorodeoxyglucose (18 F-FDG) PET in patients with Alzheimer disease, mild cognitive impairment, and healthy controls. J Geriatr Psychiatry Neurol 2010;23:18598.

21. Bourgeat P, Chetelat G, Villemagne VL, Fripp J, Raniga P, Pike $\mathrm{K}$, et al. Beta-amyloid burden in the temporal neocortex is related to hippocampal atrophy in elderly subjects without dementia. Neurology 2010;74:121-7.

22. De Santi S, Catafau A, Seibyl J, Bullich S. Robustness of 18F-Florbetaben SUVR cutoff quantification across reference regions and standards of truth. J Nucl Med 2016;57 (Suppl 2):458. 